\title{
Presencia del tiburón martillo Sphyrna zygaena (Carchariniformes: Sphyrnidae) y nuevo registro del tiburón espinudo Echinorhinus cookei (Squaliformes: Squalidae) en San Antonio, Chile central
}

\author{
José L. Brito \\ Museo Municipal de Ciencias Naturales y Arqueología de San Antonio \\ Sanfuentes 2365, Barrancas, San Antonio, Chile
}

\begin{abstract}
RESUMEN. Se documenta el registro de Sphyrna zygaena (Linnaeus, 1758), sobre la base de un ejemplar capturado en la bahía de San Antonio (333' S) y un ejemplar de Echinorhinus cookei Pietschmann, 1928, capturado al oeste de Santo Domingo (333’ S). En el primer caso, se amplía su distribución geográfica desde Arica hasta San Antonio, y en el segundo, se agrega un quinto registro para esa especie en aguas chilenas.
\end{abstract}

Palabras clave: Sphyrna zygaena, Echinorhinus cookei, Pacífico suroriental, Chile.

\section{Presence of the smooth hammerhead Sphyrna zygaena (Carchariniformes: Sphyrnidae) and new records of prickly shark Echinorhinus cookei (Squaliformes: Squalidae) in San Antonio, central Chile}

\begin{abstract}
The sighting of Sphyrna zygaena (Linnaeus, 1758) based on the capture of a specimen in the bay of San Antonio (33'35'S) and a specimen of Echinorhinus cookei Pietschmann, 1928 captured to the west of the coast of Santo Domingo ( $\left.33^{\circ} 35^{\prime} \mathrm{S}\right)$. The former case, the known geographic distribution is increased from region to the north to San Antonio, in central Chile and in the alter, a fifth sighting is noted for the species in Chilean waters, confirming its presence in the central region of Chile.
\end{abstract}

Key words: Sphyrna zygaena, Echinorhinus cookei, southeastern Pacific, Chile.

La familia Sphyrnidae Gill, 1872 está constituida por dos géneros, Sphyrna Rafinesque, 1810 y Eusphyra Gill, 1862, de los cuales solo Sphyrna está representado en el Pacífico suroriental por cinco o seis especies de aguas peruanas: Sphyrna zygaena (Linnaeus, 1758), S. lewini (Griffith \& Smith, 1834), S. mokarran (Rüppel, 1837), S. tiburo (Linnaeus, 1758) y $S$. corona Springer, 1940 , y probablemente S. media Springer, 1940 (Chirichigno, 1974).

En aguas chilenas solo se ha registrado la presencia de S. zygaena (De Buen, 1959; Bahamonde

Autor corresponsal: José L. Brito (museo_imsa@hotmail.com)
\& Pequeño, 1975; Pequeño, 1989). Esta especie es pelágica costera y/o semioceánica, y se distribuye desde la superficie hasta $50 \mathrm{~m}$ de profundidad, con una distribución anfitemperada y tropical sobre la plataforma continental de todos los continentes (Compagno, 1984; Steel, 1985). En Chile, los antecedentes sobre esta especie se limitan a registrar su presencia en aguas costeras entre Arica (1830'S) e Iquique (20¹2'S) (Fowler, 1945; Mann, 1954; Ojeda \& Avilés, 1987; Sielfeld \& Vargas, 1992).

Por su parte, la familia Echinorhinidae constituida solo por el género Echinorhinus Blainville, 1816, está representada por Echinorhinus cookei Pietschmann, 1928, que tiene una distribución 
circumpacífica, siendo registrada en Taiwán, Nueva Zelanda, Palau (Belau) e Islas de Hawai; desde el centro de California al golfo de California y Perú (11²10'S) (Compagno, 1984); frente a la costa chilena en San Antonio (3335'S) (Flores \& Rojas, 1979); isla Salas y Gómez (Parin et al., 1980), golfo de Arauco (3700'S) (Ruiz \& Fernández, 1984) y Pisagua (19¹9'S) (Meléndez \& Meneses, 1989).

En la presente nota se documenta la captura de un ejemplar de S. zygaena, que amplía su distribución geográfica en la costa del Pacífico suroriental y un quinto registro de Echinorhinus cookei en aguas chilenas, y el segundo para la costa de San Antonio, zona central.

\section{Sphyrna zygaena (Linnaeus, 1758) tiburón martillo (Fig. 1)}

Material examinado: MMSA-PE-474, cabeza de un ejemplar hembra de aproximadamente $260 \mathrm{~cm}$ de longitud total, obtenida en la bahía de San Antonio, el 19 de marzo de 1999.

Diagnosis: tiburón de cuerpo alargado, cabeza en vista dorsal ancha y con forma de martillo, con dos proyecciones aliformes laterales, en cuyos extremos se encuentran sus ojos. Contorno del margen anterior de la cabeza ondulado; quinta abertura branquial más corta que la primera; diámetro del ojo más grande que la distancia entre el ojo y el orificio nasal; margen anterior de la cabeza dividido en tres lóbulos, borde anterior de la cabeza no indentado en el centro. Boca de posición inferior, armada de dientes pequeños; la mandíbula con tres hileras de dientes visibles y un total de 26 visibles en la parte inferior. Dos aletas dorsales, la primera muy alta y sin espinas. Su coloración dorsal es café grisácea y la ventral amarillenta oscura. Las dimensiones de la cabeza en mm son: longitud preoral 108, longitud prenarial 90, alto de la primera hendidura branquial 74 , alto de la cabeza 205, largo preorbital 87, ancho interorbital 592, diámetro anteroposterior del ojo 32 , alto ojo 26 , longitud prebranquial 335, ancho de la boca 170 , ancho internarial $480 \mathrm{y}$ alto dientes centrales 7 .
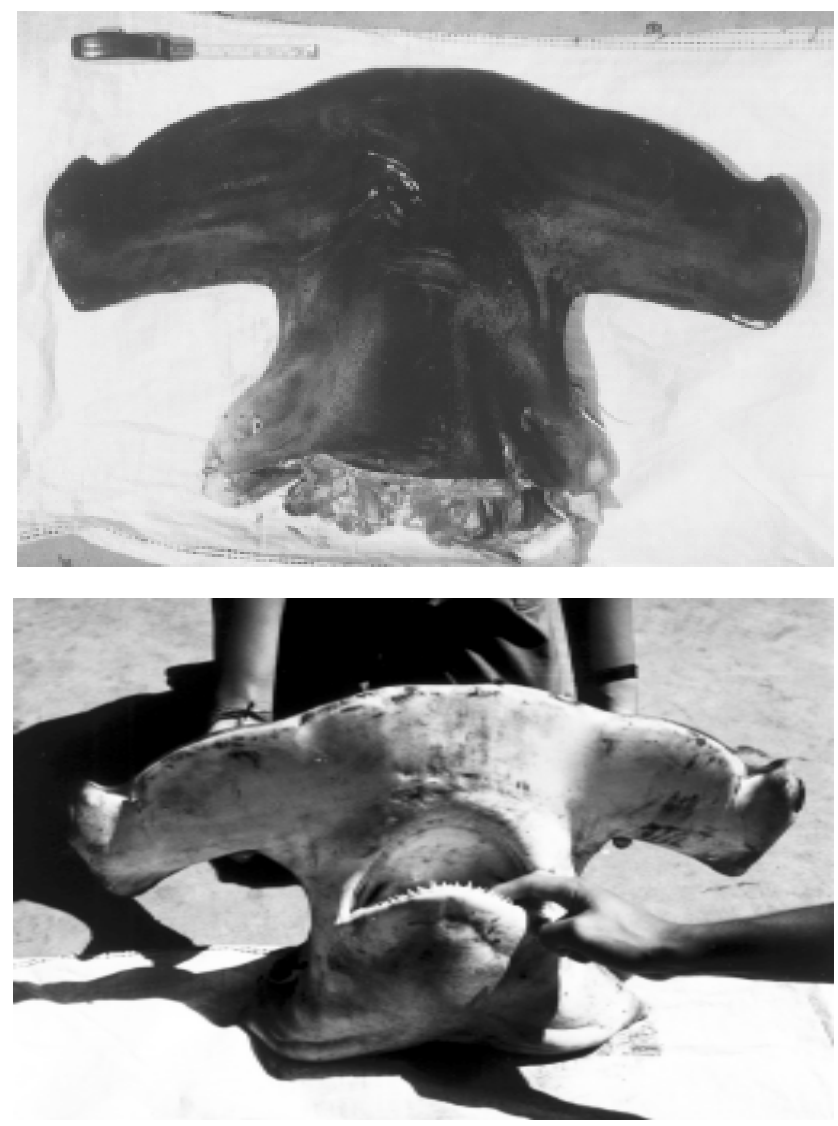

Figura 1. a) Vista dorsal, y b) vista ventral de la cabeza de Sphyrna zygaena, capturado en la bahía de San Antonio.

Figure 1. a) Dorsal view, and b) ventral view of the head of Sphyrna zygaena, captured in San Antonio bay.

Comentarios: el análisis de la cabeza y algunas fotografías del ejemplar completo obtenido en la bahía de San Antonio demuestran que se trata de S. zygaena, especie que se caracteriza porque su cráneo posee expansiones laterales, sostenidas por el alargamiento de cartílagos preorbitales y postorbitales, ubicación de los ojos, narinas muy separadas y ausencia de espiráculo. El borde anterior de la cabeza es regularmente arqueado y su margen anterior está dividido en tres lóbulos. La línea media de su cabeza es redondeada y carece de una escotadura o indentación central (Chirichigno, 1974). Tiene dientes con cúspides muy anchas y bordes lisos a suavemente serrados, con una coloración café grisácea oscura dorsalmente y más clara ventralmente. La primera aleta dorsal es moderadamente falcada y se origina a la altura de la parte posterior de la inserción de las pectorales. Dentículos del cuerpo de tamaño moderado, de base estrellada y densamente dispuestos. Esta especie alcanza una longitud entre 370 a $400 \mathrm{~cm}$ (Compagno, 1984; Sielfeld \& Vargas, 1992). 
Echinorhinus cookei Pietschmann, 1929, tiburón espinudo (Fig. 2)

Material examinado: MMSA-PE-414, un ejemplar hembra de $107 \mathrm{~cm}$ de longitud total, capturado a $208 \mathrm{~m}$ de profundidad al oeste de Santo Domingo (333's), el 7 de diciembre de 1997.

Diagnosis: tiburón de cuerpo grueso y cilíndrico. Cabeza moderadamente deprimida dorsoventralmente. La quinta y última abertura branquial abruptamente mayor que las otras; espiráculos pequeños. Boca medianamente arqueada. Dos aletas dorsales pequeñas desprovistas de espinas, ubicadas en la parte posterior del cuerpo. Dimensiones en mm: longitud total 1070, longitud de la cabeza 292, longitud preorbital, longitud precaudal 805 , distancia pre primera aleta dorsal 630, distancia segunda aleta dorsal 720, longitud prepélvica 598, espacio interdorsal 45, espacio dorsal-caudal 209 y altura pedúnculo caudal 66.

Comentarios: el ejemplar de E. cookei se pudo identificar debido a su cuerpo grueso y cilíndrico, cinco aberturas branquiales, siendo la quinta de mayor longitud; dos aletas dorsales, sin espinas, ambas de base corta y muy próximas entre sí y la primera dorsal está por detrás del origen de la aleta pélvica. La hembra analizada no presentó signos de madurez, lo que coincide con el ejemplar estudiado por Meléndez \& Meneses (1989), ya que las hembras alcanzan su madurez a los $254 \mathrm{~cm}$ de longitud (Compagno, 1984). En el estómago solo se encontraron dos vértebras de un pez óseo indeterminado.

El hallazgo de la hembra de S. zygaena en la costa de San Antonio, permite ampliar su distribución geográfica en la costa chilena, en más de trece grados de latitud hacia el sur en el Pacífico suroriental. Este registro está refrendado por un espécimen conservado que sería inmaduro, pues las hembras alcanzan su madurez a los $304 \mathrm{~cm}$ (Compagno, 1984). La presencia de dos ejemplares de jurel Trachurus symmetricus, en el contenido estomacal de esta hembra, sumada a la presencia de cardúmenes de jurel al interior de la bahía de San Antonio, el día de su captura, podría indicar que este tiburón ingresó a la bahía tras sus presas. Los registros de Caldera y San Antonio indicarían que $S$. zygaena, si bien tiene una distribución geográfica continua desde el norte hasta la zona central, es una especie escasa.

El registro de E. cookei, si bien se encuentra dentro del rango de distribución geográfica y batimétrica conocida para esta especie en la costa chilena, corresponde al quinto registro para Chile y el segundo para San Antonio, zona donde se encontró por primera vez para la costa chilena. El hallazgo de este ejemplar confirmaría lo sugerido por Ruiz \& Fernández (1984), que indican que esta especie es capturada frecuentemente por embarcaciones pesqueras, pero los ejemplares no siempre llegan a los centros de investigación.

\section{AGRADECIMIENTOS}

Al pescador artesanal Sr. Rubén Mora por la donación del ejemplar de E. cookei, al periodista $\mathrm{Sr}$. Gunter Malfert B. del Diario El Líder, por las fotografías del ejemplar de S. zygaena. Al Sr. José Segundo Brito S. por los datos aportados. Al Dr. Germán Pequeño de la Universidad Austral de Chile por la revisión crítica del manuscrito.

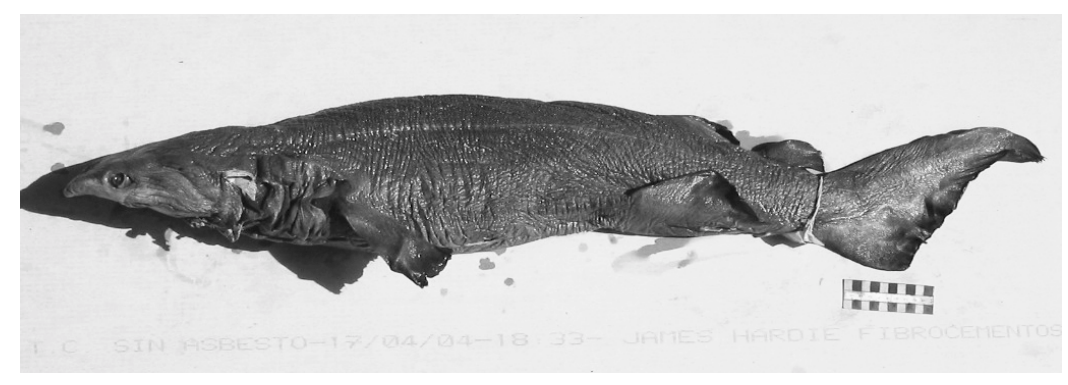

Figura 2. Ejemplar hembra de Echinorhinus cookei capturado frente a Santo Domingo.

Figure 2. Female specimen of Echinorhinus cookei captured off Santo Domingo. 


\section{REFERENCIAS}

Bahamonde, N. \& G. Pequeño. 1975. Peces de Chile. Lista Sistemática. Mus. Nac. Hist. Nat., Chile, Publ. Ocas., 21: 1-20.

Chirichigno, N. 1974. Clave para identificar los peces marinos del Perú. Inf. Inst. Mar Perú-Callao, 11: 1-387.

Compagno, L.J.V. 1984. Species catalogue. Sharks of the world. An annotated and illustrated catalogue of sharks species known to date. Part 1 . Hexanchiformes to Lamniformes. FAO Fish. Synop., 125(4): 1-125.

Flores, H. \& P. Rojas. 1979. Presencia de Echinorhinus cookei Pietschmann, 1928 frente a las costas de Chile (Pisces: Squalidae: Echinorhinidae). Invest. Mar., Valparaíso, 7(1): 4145.

Fowler, H.W. 1945. Fishes of Chile, systematic catalog. Rev. Chil. Hist. Nat., Parts I and II: 36171.

Mann, G. 1954. Vida de los peces en aguas chilenas. Ministerio de Agricultura, Instituto de Investigaciones Veterinarias y Universidad de Chile, Santiago, $324 \mathrm{pp}$.
Meléndez, R. \& D. Meneses. 1989. Tiburones del talud continental recolectados entre Arica $\left(18^{\circ} 19^{\circ} \mathrm{S}\right)$ e Isla Mocha (38 $\left.30^{\circ} \mathrm{S}\right)$, Chile. Invest. Mar., Valparaíso, 17: 3-73.

Ojeda, P. \& S. Avilés. 1987. Peces oceánicos chilenos. En: J.C. Castilla (ed.). Islas oceánicas chilenas: conocimiento científico y necesidades de investigaciones. Ediciones Universidad Católica de Chile, Santiago, pp. 247-270.

Parin, N.V., G.A. Golovan, N.P. Pakhorubov, Y.I. Sazonov \& Y.N. Shcherbachev. 1980. Fishes from the Nazca and Salas y Gómez underwater ridges collected in cruise of R/V "Ikhtiandr". En: N.V. Parin (ed.). Fishes of the open ocean. Academy of Sciences of the USSR; PP. Shirshov Institute of Oceanology, Moscow, pp. 5-18.

Pequeño, G. 1989. Peces de Chile. Lista sistemática revisada y comentada. Rev. Biol. Mar., Valparaíso, 24(2): 1-132.

Ruiz, V. \& J. Fernández. 1984. Primer registro de Echinorhinus cookei Pietschmann, 1928 (Pisces: Squaliformes: Squalidae) para Chile. Bol. Soc. Biol. Concepción, 55: 175-176.

Sielfeld, W. \& M. Vargas. 1992. Tiburones y rayas del Pacífico sur oriental. Departamento de Ciencias del Mar, Universidad Arturo Prat, Iquique, 102 pp.

Steel, R. 1985. Sharks of the world. Blandford Press, New York, 192 pp. 


\section{IN MEMORIAM}

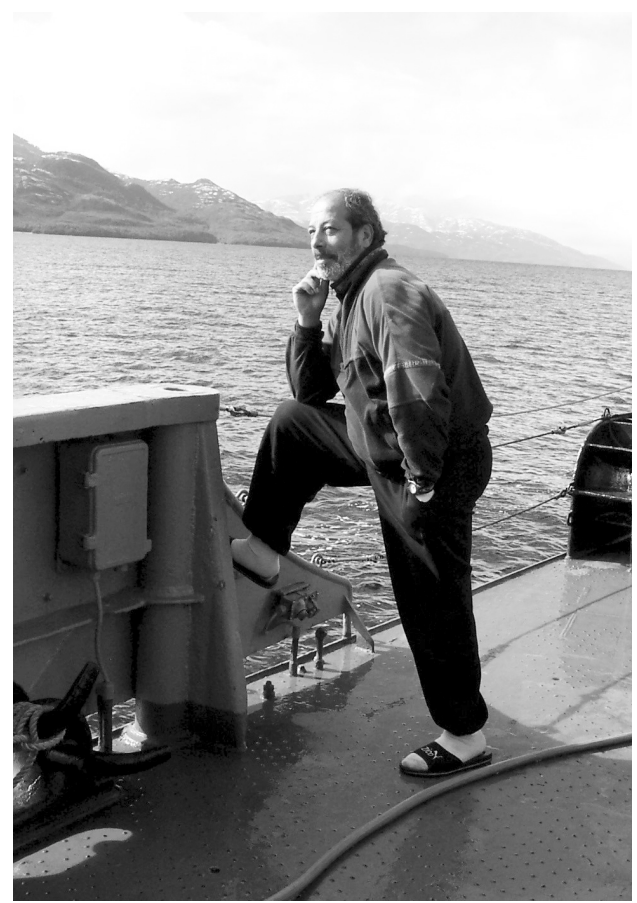

\section{Reinaldo Rehhof Díaz}

(1951 - 2004)

Reinaldo Rehhof, Técnico Muestreador de la Escuela de Ciencias del Mar de la Pontificia Universidad Católica de Valparaíso, falleció el 22 de mayo 2004 en Puerto Aysén, durante la noche mientras dormía a la espera de embarcarse para realizar un nuevo trabajo oceanográfico en Puerto Chacabuco. Creo que falleció como a él le habría gustado, en su trabajo, al que amaba mucho y del cual estaba orgulloso por su desempeño, situación que era reconocida por todos los que le conocimos.

Reinaldo fue una persona llena de vida y energía, siempre colaborando, nunca dejando algo por hacer y de hacer bien. Para él no habían excusas, la perfección era su norte.

Para los que trabajamos directamente con él, teníamos una fe ciega en la calidad del trabajo que haría y ya no eran necesarias las instrucciones, solo bastaba decir que queríamos algo y él lo resolvía.

Espero que nuestra muy querida pareja de técnicos muestreadores fallecidos, Noé primero y ahora Reinaldo, se hayan reunido en el más allá para continuar con su gran compañerismo y amistad, que siempre los caracterizaron.

Reinaldo, descansa en paz.

NeLSON SiLVA 


\section{INSTRUCCIONES A LOS AUTORES}

La Revista Investigaciones Marinas publica trabajos inéditos y originales en ciencia y tecnología del mar, en español o inglés, dando preferencia a los realizados en el Océano Pacífico Suroriental y Océano Austral.

El Comité Editorial de la Revista, asesorado por un mínimo de dos evaluadores, se reserva el derecho de revisar críticamente los trabajos y decidir su publicación.

Los autores deberán enviar el trabajo en cuatro ejemplares, escrito en formato carta a espacio y medio. Además, deben adjuntar un diskette o CD con el trabajo en formato digital. El texto en Microsoft Word. Las figuras (Corel Draw o JPG) y tablas (Excel), con leyendas en español e inglés.

\section{Dirección del Editor:}

Revista Investigaciones Marinas

Casilla 1020 - Valparaíso, Chile

Fono: 56-32-274258. Fax: 56-32-274206

E-mail: investmar@ucv.cl

Para la presentación del documento en español se debe seguir el siguiente esquema:

Título: Breve y descriptivo, escrito en español e inglés. Además, indicar un título resumido no mayor de 50 caracteres, para el encabezamiento de las páginas.

Autores: Indicar nombre, primer apellido, institución, dirección y correo electrónico.

Resumen: Con un máximo de 250 palabras, que describa claramente el problema, los principales resultados y posibles conclusiones. Agregar un máximo de seis palabras clave en orden de importancia.

Abstract: Traducir al inglés el resumen y las palabras clave.

\section{Introducción}

Materiales y Métodos

Resultados

Discusión
Conclusiones (opcional)

Agradecimientos (opcional)

Referencias: Indicar solamente los trabajos mencionados en el texto, ordenados por orden alfabético de los autores. La inicial del nombre y apellido de los autores se escribirán con letras mayúsculas-minúsculas y en negrita. Si la referencia tiene más de un autor, a partir del segundo, la inicial del nombre precederá al apellido y los autores irán separados por coma.

a) Las referencias de artículos deben seguir el orden siguiente: autor(es), año de publicación, título del artículo, nombre abreviado de la revista (según el trabajo World List of Scientific Periodicals), volumen y número entre paréntesis, primera y última página.

Astthorsson, O. \& A. Gislason. 1991. Seasonal abundance and distribution of Caridea larvae in lsafjord-deep, north-west Iceland. J. Plankton Res., 13(1): 91-102.

b) Las referencias de libros deben indicar: autor(es), año de publicación, título del libro, editorial, ciudad, páginas.

Neshyba, S. 1987. Oceanography. Perspectives on a fluid earth. John Wiley \& Sons, New York, 506 pp.

c) Las referencias de artículos publicados en libros deben señalar: autor(es), año de publicación, título del artículo, editor(es), nombre del libro, editorial, ciudad, páginas.

Hokanson, K. \& C. Kleiner. 1974. Effects of constant and rising temperatures on survival and development rates of embryonic and larval yellow perch Perca flavescens (Mitchill). En: J. Blaxter (ed.). The early life history of fish. Springer-Verlag, Berlin, pp. 437-448.

d) Las referencias de artículos publicados en Internet deben señalar: autor(es), año de publicación, título del artículo, sito web, fecha de revisión. 
Walker, J.R. 1997. MLA-Style citations of Internet sources. Página www [http://www.cas.usf.edu/ english/walker/janice.html]. Revisado: 27 de enero 1999.

e) Las referencias de artículos o libros publicados en CD-ROM deben señalar: autor(es), año de publicación, (CD-Rom), título del artículo o libro, editorial, ciudad.

Retamal, M.A. 2000. (CD-ROM). Decápodos de Chile. ETI-Universidad de Concepción. Springer-Verlag, Berlin.

\section{Instrucciones para el texto del documento}

1. Los trabajos no deben exceder las 30 páginas incluidas tablas y figuras; la ubicación de éstas debe indicarse en el margen de las páginas del texto.

2. Las unidades deben expresarse de acuerdo al Sistema Internacional de Unidades. Si es necesario, podrán utilizarse otras, cuyo significado debe ser explicado en la primera oportunidad en que se utilicen.

3. Las referencias bibliográficas deben indicarse por el apellido del autor y el año de publicación, según sea un autor, dos autores, más de dos autores o varios trabajos de un autor en un mismo año (Alvarez, 1986; Johnson \& Smith, 1981; Muñoz et al., 1977; Palmer, 1989a, 1989b).

4. Citar solamente los trabajos publicados o en prensa. En este último caso, indique en la lista de referencias el nombre del autor(es), el título y la revista, seguida de las palabras (en prensa). Las comunicaciones personales se citarán sólo en el texto como sigue (autor, com. pers.). Ejemplo (S. Paredes, com. pers.).

\section{Instrucciones para las figuras y tablas}

1. Los gráficos, mapas, esquemas, dibujos o fotografías deben denominarse Figura y en el texto se abreviarán como Fig. Las figuras se enumerarán en forma correlativa con números árabes; las leyendas deben ser autoexplicativas y escritas en hoja aparte. Las figuras se deben enviar en papel blanco de buena calidad. Las figuras, letras y símbolos deben ser de un tamaño que no requieran más de tres reducciones, para ajustarse al formato de la página y asegurar que los símbolos no sean inferiores a 1,5 mm de alto.

2. Las Tablas se enumerarán en forma correlativa con números árabes; las leyendas deben ser autoexplicativas y ubicadas en su parte superior. El encabezamiento de cada columna debe expresar claramente su contenido y unidades de medición.

3. Las fotografías deberán ser enviadas con sus respectivos negativos, considerando un buen rango de tonos y contrastes.

\section{Notas Científicas}

Los trabajos cortos sobre un tema específico, que describan métodos o resultados, se publicarán como Notas Científicas. Las Notas deben contener un resumen (máximo de 200 palabras) y palabras claves, escritos en castellano e inglés. La introducción, metodología, resultados y discusión debe ser escrita en forma continua, sin individualizar los subtítulos, en una sola sección. La extensión del manuscrito será inferior a nueve páginas incluyendo las figuras.

Pruebas de imprenta y separatas: Las pruebas de imprenta serán normalmente revisadas por los autores, salvo que el Comité Editorial lo estime innecesario. De cada artículo se entregarán veinte separatas; no obstante, los autores podrán solicitar copias adicionales a precio de costo.

Costos de impresión: Los autores o sus instituciones deberán cancelar US\$ 10 por página impresa y US\$ 80 por página en colores, para cubrir parte de los costos de impresión y distribución. Estos valores deberán ser cancelados cuando el autor devuelva las pruebas de imprenta de su artículo. 


\section{INSTRUCTIONS FOR AUTHORS}

The journal Investigaciones Marinas publishes previously unpublished, original MSS in science and technology related to the sea, in Spanish or English, with preference for topics related to the Southeastern Pacific Ocean and Southern Ocean .

The Editorial Committee of the Journal, advised by a minimum of two reviewers, reserves the right to critically review all MSS and decide on their acceptability for publication.

Authors should send four copies of the MS, on letter size paper and spacing of 1.5 format. All manuscripts should also be provided in a diskette as a Microsoft Word document. Figures (Corel Draw or JPG) and Tables (Excel) with legends, both Spanish and English.

\section{Editor's address:}

Revista Investigaciones Marinas

P.O. Box 1020 - Valparaíso, Chile

Phone: 56- 32-274258. Fax: 56-32-274206

E-mail: investmar@ucv.cl

\section{Presentation of manuscripts in English}

Title: Brief and self-descriptive, written both in English and Spanish. A usually shorter running title not exceeding 50 characters should be included for page headings.

Authors: Name and surname, institution address and e-mail.

Abstract: Short and precise, both in English and Spanish. Use no more than 250 words, describing the subject of study, main results and conclusions. A maximum of six key words may be added.

\section{Introduction}

Material and Methods

\section{Results}

Discussion

Conclusions (optional)

Acknowledgements (optional)
References: Only references cited in the paper should be included, by alphabetical order of authors. First name initial and surname should be written in bold capital-lower case letters. If more than one authors, starting with the second one, name initial will precede the surname, and authors will be separated by coma.

a) References to articles should include: author(s), year of publication, article title, journal abbreviations (following the World List of Scientific Periodicals), volume, number in parenthesis, first and last page.

Astthorsson, O. \& A. Gislason. 1991. Seasonal abundance and distribution of Caridea larvae in lsafjord-deep, north-west Iceland. J. Plankton Res., 13(1): 91-102.

b) Book references should follow: author(s), year of publication, book title, publisher, city, pages.

Neshyba, S. 1987. Oceanography. Perspectives on a fluid earth. John Wiley \& Sons, New York, 506 pp.

c) References to articles published in books should indicate: author(s), year of publication, article title, editor(s), name of the book, publisher, city, pages.

Hokanson, K. \& C. Kleiner. 1974. Effects of constant and rising temperatures on survival and development rates of embryonic and larval yellow perch Perca flavescens (Mitchill). In: J. Blaxter (ed.). The early life history of fish. Springer-Verlag, Berlin, pp. 437-448.

d) References to articles published on the Internet should indicate: author(s), year of publication, article title, website, date of revision.

Walker, J.R. 1997. MLA-Style citations of Internet sources. Page www [http://www.cas.usf.edu/ english/walker/janice.html]. Revised: 27 Juanuary 1997.

e) References to articles or books published in CDRom should indicate: author(s), year of publication, (CD-ROM), article or book titles, editorial, city. 
Retamal, M.A. 2000. (CD-ROM). Decápodos de Chile. ETI-Universidad de Concepción. Springer-Verlag, Berlin.

\section{Instructions for the text of the manuscript}

1. Papers should not exceed 30 pages, including tables and figures; location of these should be indicated at the margin of text pages.

2. Units should be expressed according to the International System of Units. If necessary, others could be used, but its meaning should be explained in the text when used for the first time.

3 . References should be indicated by the author's surname and year of publication, as follows in the case of one, two or more authors or several papers of the same author in the same year, respectively (Alvarez, 1986; Johnson \& Smith, 1981; Muñoz et al., 1977; Palmer, 1989a, 1989b).

4. Citations should only address published or papers in press. In the latter case, the full reference should be included in the references list, followed by the words (in press). Personal communications are accepted only in the text (author, pers. com.), as follows (J. Smith, pers. com.).

\section{Instructions for figures and tables}

1. Graphs, maps, schemes, drawings or photographs should be named as figures and abbreviated as Fig. in the text. All Figures shall be numbered consecutively with Arabic numerals; legends should be self-explaining and written on a separate sheet. Figures should be sent on good quality white paper. Figures, letters and symbols should be sized so that they would not require reduction of more than $3 \mathrm{X}$ to fit the journal's page format, and ensuring that the smallest one would be least $1.5 \mathrm{~mm}$ high when reduced.

2. Tables should be consecutively numbered with Arabic numerals; legends should be self-explaining and located in its upper part. Heading of each column should clearly express its content and measurement units.

3. Photographs should be sent with their negatives, considering an acceptable range of tones and contrasts.

\section{Short Communications}

Short topical papers, describing methods and results, will be published as Short Communications. The Communications should include an abstract (maximum 200 words) and the key words, both in English and Spanish. The introduction, methodology, results and discussion should be presented in a single section. The total length of manuscripts should not exceed nine pages including the figures.

Proofs and reprints: Authors themselves shall normally review proofs, unless the Editorial Board considers this unnecessary. Twenty reprints will be sent to the author free or charge. Additional reprints may be purchased at minimum cost by the authors.

Page charges: The costs of printing are US $\$ 10$ per printed page and US $\$ 80$ per printed colour page, to cover part of the publication costs. This cost should be paid by the authors or their institutions when returning the corrected proof. 